\title{
Germanica
}

\section{Monika Maron Endmoränen. Le vieillissement comme crise : entre expérience de la perte et aspiration à un nouveau départ}

Monika Maron Endmoränen. Alternsprozess als Krise: Zwischen Erfahrung des

Verlusts und Sehnsucht nach einem Neuanfang

Monika Maron Endmoränen. Ageing process as crisis: between experience of

loss and aspiration for a new beginning

\section{Emmanuelle Aurenche-Beau}

\section{OpenEdition}

Journals

Édition électronique

URL : http://journals.openedition.org/germanica/1556

DOI : 10.4000/germanica.1556

ISSN : 2107-0784

Éditeur

Université de Lille

Édition imprimée

Date de publication : 10 juillet 2012

Pagination : 33-45

ISBN : 9782913857292

ISSN : 0984-2632

Référence électronique

Emmanuelle Aurenche-Beau, « Monika Maron Endmoränen. Le vieillissement comme crise : entre expérience de la perte et aspiration à un nouveau départ », Germanica [En ligne], 50 | 2012, mis en ligne le 17 juillet 2012, consulté le 06 octobre 2020. URL : http://journals.openedition.org/germanica/1556 ; DOI : https://doi.org/10.4000/germanica.1556

Ce document a été généré automatiquement le 6 octobre 2020.

(c) Tous droits réservés 


\title{
Monika Maron Endmoränen. Le vieillissement comme crise : entre expérience de la perte et aspiration à un nouveau départ
}

\author{
Monika Maron Endmoränen. Alternsprozess als Krise: Zwischen Erfahrung des \\ Verlusts und Sehnsucht nach einem Neuanfang \\ Monika Maron Endmoränen. Ageing process as crisis: between experience of \\ loss and aspiration for a new beginning
}

Emmanuelle Aurenche-Beau

\begin{abstract}
Avec l'allongement de la durée de la vie, le thème de la vieillesse et du vieillissement est un thème qui tend à prendre une place croissante dans la littérature de nos sociétés occidentales, aux États-Unis par exemple avec Philipp Roth et ses romans centrés sur des personnages masculins âgés, en France avec les textes de Noëlle Châtelet (La Femme coquelicot) ou d'Annie Ernaux (Une femme) pour n'en citer que quelques-uns. La littérature de langue allemande évidemment n'est pas en reste, que l'on pense aux romans de Martin Walser, Der Augenblick der Liebe ou Der Lebenslauf der Liebe par exemple, qui mettent l'accent sur le désir amoureux chez des personnages vieillissants. Le personnage principal du roman de Monika Maron Endmoränen ${ }^{1}$, quant à lui, est une femme entre cinquante et soixante ans, Johanna, qui relate à la première personne, un peu à la manière d'un journal intime, les quelques semaines qu'elle passe seule, à la fin d'un été, dans sa maison de campagne de Basekow, dans un paysage du Brandebourg marqué par l'érosion glaciaire qui explique l'allusion du titre à des «moraines ». Mais ces «moraines» peuvent aussi être comprises dans un sens métaphorique comme l'accumulation des souvenirs qu'il s'agit, à l'aube d'une nouvelle phase de vie, de reconsidérer et de réévaluer. Johanna, en effet, traverse une crise qui comprend à la fois des aspects politico-sociaux, liés à la fin de la RDA et à la réunification qui ont eu des conséquences directes sur sa vie professionnelle, et des aspects plus personnels : c'est tout son rapport à elle-même, à ses projets, à ses proches, à sa place et à son rôle
\end{abstract}


dans la société qu'elle a à repenser. Nous tenterons donc de montrer comment le roman décrit cette période critique de la vie de Johanna entre expérience de la perte, expérience du retrait et possibilité d'un nouveau départ.

\section{La vieillesse comme expérience de la perte}

2 Comme les autres romans consacrés au vieillissement, le roman de Monika Maron évoque tout d'abord les transformations du corps, du visage et la perte de la capacité à séduire. Johanna observe ainsi sur sa peau les traces du vieillissement ${ }^{2}$, évoque ses cheveux qui grisonnent ${ }^{3}$, son "combat désespéré contre les cheveux, la peau et la chair $»^{4}$, son visage qui se transforme inexorablement en un visage de vieille femme ${ }^{5}$. Elle ne supporte plus de se voir nue, évite les douches et ne se baigne plus que sous une "couverture de mousse » qui la dissimule à elle-même ${ }^{6}$. Elle évoque également les regards des hommes qui la "neutralisent» et qui lui signifient qu'elle n'est plus désirable ${ }^{7}$. Et elle affirme que "la seule passion naturelle que l'âge puisse encore permettre est les petits-enfants $»^{8}$, mais y croit-elle vraiment ? Elle précise en effet que " c'est la nuit et qu'elle voit peut-être les choses de manière trop noire ${ }^{9}$ et ne semble pas tout à fait convaincue par les propos de son amie Elli qui, dans un autre contexte, affirme (par dépit?) que l'amour n'est que le rêve (illusoire) d'un bonheur paradisiaque génétiquement programmé ${ }^{10}$ et qui ne comprend pas ceux qui courent encore après l'amour une fois passé le temps de la jeunesse ${ }^{11}$.

3 À ces descriptions des manifestations physiques du déclin lié à l'âge et à ces questionnements douloureux sur la poursuite d'une vie amoureuse s'ajoute pour Johanna une profonde remise en question de sa valeur sociale qui se manifeste tout d'abord par une crise du sens de son travail. Son projet, au cours de ces semaines qu'elle a décidé de passer seule à Basekow, est d'écrire une biographie de Wilhelmine Enke, une des maîtresses de Frédéric Guillaume II ; or elle ne sait plus pourquoi elle a proposé ce sujet à son éditeur alors qu'elle le lui avait pourtant présenté comme une " contribution originale à l'histoire de la Prusse ${ }^{12}$ et ne parvient pas à se mettre au travail. Après des études de germanistique, Johanna avait, du temps de la RDA, travaillé comme rédactrice, rédigeant des textes de présentation destinés à des pochettes de disques ainsi que des notices biographiques, des préfaces ou des postfaces, et le sens de son travail résidait pour elle dans le fait de dissimuler des «messages » dans ses textes. Avec la chute du mur, cette activité a perdu tout son sens et, sans être encore une vieille femme à la charge de la société ${ }^{13}$, Johanna se sent désormais inutile ${ }^{14}$. Tout se passe en réalité comme si les bouleversements liés à la fin de la RDA avaient accéléré et exacerbé un processus inexorable, indissociable de l'expérience du vieillissement, une longue vie professionnelle pouvant difficilement ne pas connaître d'évolution dans ses conditions d'exercice. Christian P., l'ami ouest-allemand à qui Johanna écrit plusieurs lettres au cours de ces semaines de solitude, fait d'ailleurs une expérience similaire dans un contexte tout à fait différent. Il se voit en effet mettre dans un placard ou plutôt dans un grenier par la maison d'édition dans laquelle il travaille, car l'évolution de la société fait que ce ne sont plus désormais les ouvrages de sciences humaines, mais les livres traitant de questions de bioéthique ou de médias qui ont le vent en poupe $\mathrm{e}^{15}$.

4 Johanna doit en outre repenser sa place auprès de ses proches. Son mari, Achim, spécialiste de Kleist, semble désormais entièrement absorbé par la rédaction de projets et de demandes de financements pour pouvoir poursuivre ses recherches dans le 
contexte de l'Allemagne réunifiée où le soutien de la recherche n'est plus assuré en totalité par l'État comme du temps de la RDA, à moins qu'il n'ait une liaison, comme elle en fait l'hypothèse lors d'une conversation téléphonique où elle a l'impression qu'il n'est pas seul. La communication entre eux semble très réduite et Johanna se rend compte que ce n'est plus à Achim qu'elle s'adresse quand une réflexion lui vient - elle se surprend à se confier à son ami Christian P. plutôt qu'à son mari qui n'est plus pour elle qu'un « dos ».

Les relations avec sa fille Laura, âgée de 27 ans, semblent également se distendre. Cette dernière est fascinée par l'Amérique - elle revient d'un long voyage aux États-Unis et ne rêve que d'y retourner pour s'y installer définitivement. L'Allemagne lui apparait comme un pays de gens qui manquent de fierté, car ils n'assument pas leurs défaites ${ }^{16}$ et Johanna craint qu'elle ne vende la maison de Basekow quand Achim et elle ne seront plus là. Indépendamment du fait que Laura, qui est maintenant adulte, souhaite faire sa vie ailleurs, Johanna souffre aussi du fait que sa fille est peu encline à se confier à elle et qu'elle doit se surveiller pour ne pas lui poser trop de questions qui risqueraient de l'agacer et de la fermer davantage encore.

C'est donc dans un certain isolement que se retrouve Johanna et elle décrit avec beaucoup de finesse ce qu'elle éprouve entre vide, ennui et apathie. Son rapport au temps notamment est en crise. Son rapport aux saisons en particulier s'est modifié, elle se sent dans une proximité nouvelle avec l'automne et éprouve depuis peu non plus un sentiment de "perte», d'« impudence», de «douleur», mais un sentiment de "soulagement» quand il arrive ${ }^{17}$, c'est d'ailleurs sur cette remarque que le livre s'ouvre. Johanna souligne aussi que son rapport à l'avenir a évolué, l'avenir ne lui apparaît plus comme le temps des possibles et des projets, il n'est plus que « continuation fatigante de ce qui a été $~^{18}$. Elle semble faire siens les propos de son ami Christian P. qui note qu'à leur âge, toutes les grandes décisions de la vie sont prises : «Le métier, le nombre d'enfants, le lieu de vie, tout est définitif $»^{19}$. Et le temps qui reste semble trop court pour corriger les erreurs commises. Ce temps qui reste ${ }^{20}$ et qui n'est plus tendu vers l'avenir semble vide et Johanna fait l'expérience de l'ennui. Elle n'a plus envie de rien faire et doute qu'elle ait envie de prolonger une telle vie si la possibilité lui en était donnée ${ }^{21}$. Elle ne parvient pas à travailler à la biographie qu'elle doit écrire ${ }^{22}$, elle a perdu toute motivation - il ne lui reste que la motivation financière - et elle ne retrouve pas les raisons qui l'avaient poussée à s'intéresser au personnage de Wilhelmine $E_{n k e}{ }^{23}$. Elle se décrit comme étant dans un état de léthargie, d'apathie qu'elle évoque en employant les termes de «Stumpfsinn» (EM, 49; 53), de «Schläfrigkeit» (EM, 183) dont rien ne semble pouvoir la faire sortir, dans lequel elle semble même se complaire ${ }^{24}$. Elle finit même par tomber dans une sorte de résignation chronique, comme le lui fait remarquer Igor, le jeune galeriste russe ami de sa voisine Karoline ${ }^{25}$. Johanna, en effet, semble tout entière tournée vers le passé, ruminant des regrets quant à ce qu'elle considère comme les moments manqués de sa vie. Elle se reproche notamment de ne pas avoir su répondre à l'amitié que lui portait Irene, son amie d'enfance infirme, dont elle apprend trop tard qu'elle était la seule personne qu'elle aurait souhaité voir avant sa mort. Elle se reproche aussi de n'avoir pas su saisir la chance de nouveau départ que lui offrait la fin de la RDA et les changements qui en ont découlé pour commencer une nouvelle vie, à l'instar d'un collègue d'Achim, spécialiste de Tieck, devenu ministre ou d'un de ses collègues, secrétaire du Parti reconverti dans les pompes funèbres ${ }^{26}$ : "À l'époque j'aurais sans doute dû arrêter d'écrire des biographies ${ }^{27}$ » et elle se met alors à rêver de ce qu'elle aurait pu faire : 
«devenir fleuriste ou femme politique, représentante en assurances ou fonder une entreprise de pompes funèbres $»^{28}$.

\section{Le temps du retrait, de l'écriture et de la réflexion}

7 Le temps présent des quelques semaines qu'elle passe à Basedow en tout cas est décrit comme un temps qui ne passe pas, qu'elle tente de "tuer » en jardinant, en bricolant, en lisant, en téléphonant, en regardant des séries télé, en observant la nature qui l'entoure ${ }^{29}$. Pourtant, de ce temps de retrait, de ce temps perdu semble émerger peu à peu une réflexion sur sa vie et plus généralement sur la vie et l'expérience du vieillissement. Ce n'est sans doute pas un hasard si elle compare cette épreuve à une traversée, à la situation de quelqu'un qui serait «sur un bateau au beau milieu de la mer obscure, coupé de tous et de tout ce qui aurait pu faire office de repère $»^{30}$. Tout se passe d'ailleurs comme si le livre était pour ainsi dire le journal de cette traversée. Même si les différents chapitres ne sont pas datés avec précision, ils comportent tout de même des indications temporelles qui indiquent qu'on a à faire à un déroulement chronologique ${ }^{31}$ avec une alternance de passages réflexifs et de passages plus narratifs où Johanna raconte tout simplement ce à quoi elle passe ses journées, insérant même les lettres qu'elle écrit et qu'elle reçoit ainsi que ses notes sur Wilhelmine Enke ${ }^{32}$. Grâce à des phénomènes de réflexion (au sens optique du terme), produits par l'évocation de ses rencontres, de ses conversations, de ses échanges épistolaires émerge peu à peu un questionnement sur la vie et sur le bonheur : qu'est-ce qu'une vie heureuse, qu'est-ce qu'une vie qui a du sens, qu'est-ce qu'une vie pleinement vécue? peut-on être heureux malgré des manques? peut-on compenser ces manques? Johanna souligne d'ailleurs elle-même à quel point le passage par le "récit ", le fait de raconter ce qu'elle vit a toujours été déterminant pour elle : «Je menais une double vie, une vraie vie et une vie racontée, l'une se distinguant à peine de l'autre; je comprenais ce que j'avais vécu seulement en le racontant et en imaginant ce qui se serait passé si j'avais fait le choix inverse $\aleph^{33}$. Quelle que soit la forme qu'il prenne, que ce soit une forme orale (les interminables conversations avec son amie Elli, par exemple, dont Achim ne comprend pas l'intérêt ${ }^{34}$ !) ou une forme écrite adressée, les lettres, ou plus solitaire, le journal luimême ou encore une forme indirecte, l'écriture de biographies, le " récit » est ce qui lui permet d'avancer dans ses réflexions - le critique Lutz Hagestedt va même jusqu'à affirmer à propos de la biographie de Wilhelmine Enke: «Il (le lecteur) doit comprendre que ce livre n'est pas seulement le problème, mais aussi la solution du problème $»^{35}$.

On pourrait, en adoptant un autre point de vue, faire l'hypothèse que l'auteure a " construit " les personnages secondaires du roman de manière à mettre en scène les questionnements de Johanna. La vie d'Irene, par exemple, son amie d'enfance qui, handicapée, est restée célibataire et a vécu toute sa vie avec sa mère peut poser la question de la place du bonheur dans une vie qu'elle résume ainsi: "une vie brève, gâtée par une colonne vertébrale déformée, sans amour et sans enfants ${ }^{36}$. Sa passion pour les études tchèques (Irene était chercheuse en slavistique) a-t-elle pu compenser ces souffrances et ces manques? Il en va de même pour son amie Elli, journaliste, et sa voisine Karoline, peintre, qui, pour des raisons différentes, vivent seules et n'ont pas d'enfants non plus : la réussite professionnelle, l'art, la peinture, la réalisation d'une œuvre et la reconnaissance sociale qui en résulte peuvent-ils donner sens à une vie ? 
$\mathrm{Ou}$, si l'on pose la question sous une autre forme, que doit-il rester d'une vie pour que cette vie puisse être considérée comme réussie: un enfant, une œuvre scientifique, artistique? Le plus important est-il d'ailleurs ce qui reste d'une vie ou le sentiment d'avoir pleinement vécu sa vie? Le personnage d'Elli qui a quitté la RDA pour aller vivre à l'ouest (ce que Johanna n'a pas fait, retenue, dit-elle, par son mari et par sa fill ${ }^{37}$ ) pourrait donner le sentiment qu'une vie pleinement vécue serait une vie où l'on a su prendre des risques, oser un départ. Elle note ainsi à propos d'Elli : « Pendant tout le temps que je connus Elli, je la considérai toujours comme plus adulte et comme possédant une plus grande intelligence de la vie que moi, surtout comme plus indépendante. Au moment où elle est partie pour Kreuzberg, j'ai pensé que, dans la vie, Elli prenait toujours la bonne décision et moi la mauvaise ${ }^{38}$. Elle envie d'ailleurs peutêtre aussi secrètement sa fille, qui envisage de quitter l'Allemagne pour s'installer définitivement aux États-Unis et admire en tout cas son fort tempérament et son caractère volontaire ${ }^{39}$.

D'autres personnages du roman pourraient donner à penser qu'une vie pleinement vécue est une vie où l'on vit jusqu'au bout ses passions, à l'instar de Friedel Wolgast, une voisine âgée qui va jusqu'au bout dans la haine qu'elle éprouve envers son voisin de l'ouest ${ }^{40}$, ou, dans un autre registre, de la femme de Christian P. qui, après des années de mariage, décide de se séparer de son mari pour vivre avec l'homme dont elle est tombée amoureuse ${ }^{41}$. Une vie réussie ne serait-elle pas, plus sagement, une vie dans laquelle on a su vivre avec ce qui se présentait à soi, c'est-à-dire aussi bien saisir les chances qui apparaissaient qu'accepter les limites, les manques et surmonter les crises? Telle pourrait être la «leçon» à tirer de la vie de Wilhelmine Enke dont Johanna, comme on l'a vu, tente d'écrire la biographie et dont elle sent confusément qu'elle a une fonction de "parabole", même si elle ne parvient pas à en retrouver le sens : "J'avais trouvé, à l'époque, dans le destin de Wilhelmine Enke, future comtesse Lichtenau, une parabole qui ne semblait pas seulement contenir une réponse aux égarements de ma propre vie, mais aussi quelque chose d'exemplaire, une déduction en retour ou une autre sorte de logique complexe qui ne m'était plus accessible. $»^{42}$ Malgré la distance temporelle et les différences de contexte, plusieurs points de la vie de Wilhelmine Enke en effet peuvent intéresser et rejoindre Johanna. On pourrait citer tout d'abord leur intérêt commun pour les messages indirects comme moyen de « jouer » avec le pouvoir : de même que Johanna par les messages qu'elle glisse dans ses textes peut contribuer, à son échelle, à une action de subversion du discours officiel, de même Wilhelmine Enke peut-elle, en manipulant les esprits, tenter de réduire l'influence des rose-croix sur le roi et sur la politique prussienne ${ }^{43}$. On pourrait remarquer également que plusieurs éléments de la vie de Wilhelmine Enke rejoignent la question du nouveau départ. Cette dernière a su, en effet, par exemple, saisir la chance qui lui a été offerte quand le roi l'a remarquée malgré sa médiocre condition sociale et lui a donné la possibilité de recevoir une éducation culturelle et artistique complète ${ }^{44}$. Wilhelmine Enke, de plus, est restée jusqu'à sa mort, même lorsqu'elle n'était plus sa maîtresse, la confidente et l'amie du roi : "La véritable provocation ne résidait-elle pas précisément dans le fait que cette maîtresse se soit, pour ainsi dire, survécu à elle-même sans perdre son rang et son influence ? $»^{45}$ Wilhelmine Enke, enfin, a commencé, à cinquante ans, une nouvelle vie en épousant un homme beaucoup plus jeune qu'elle (un auteur de théâtre de vingt-six ans, Franz von Holbein ${ }^{46}$ ). En tout cela, Wilhelmine Enke peut donc apparaitre à Johanna comme une sorte de « modèle ». 


\section{Vieillesse et possibilité d'un nouveau départ?}

10 La fin du roman contient, en tout cas, différents indices semblant suggérer que Johanna, comme Wilhelmine Enke, est prête à prendre elle aussi un nouveau départ : après une phase "dépressive » de lamentation sur les pertes liées au vieillissement, suivie d'une phase réflexive, on arriverait ainsi à une phase plus active. Johanna, en effet, décide de quitter sa retraite de Basekow pour rentrer à Berlin. Elle semble avoir de nouveaux projets, notamment celui d'une biographie de Natalia Timofojevna, la princesse russe dont lui a parlé Igor, le jeune galeriste ami de Karoline, dont la vie, remplie, comme celle de Wilhelmine Enke, de moments de crise et de nouveaux départs, est un véritable roman : fille d'une famille noble de Moscou qui a émigré en Allemagne au moment de la Révolution russe, elle a fréquenté les milieux artistiques de Berlin qu'elle quitte à l'arrivée au pouvoir de Hitler. En exil au Mexique, elle épouse un communiste allemand avec qui elle vit ensuite en RDA où il travaille pour la police politique. Après sa mort et après la fin de l'Union soviétique, elle retourne en Russie où elle se retrouve à la tête d'une gigantesque fortune grâce à laquelle Igor espère créer une fondation et un musée destiné à faire connaître la peinture russe à Berlin. Le livre suivant de Monika Maron, Ach Glück ${ }^{47}$, qui montre Johanna travaillant dans la galerie d'Igor à Berlin et entamant une correspondance avec Natalia Timofojevna, qu'elle décidera de rejoindre au Mexique pour l'aider à retrouver les traces de Leonora Carrington, une artiste peintre et écrivaine surréaliste à la vie mouvementée, amie notamment de Max Ernst, qu'elle a fréquentée pendant sa jeunesse ${ }^{48}$, semble confirmer la réalité de ce nouveau départ, assez mal perçu d'ailleurs par la critique de la part d'une femme vieillissante ${ }^{49}$.

11 Johanna, d'autre part, semble être parvenue à une plus grande autonomie vis-à-vis de ses proches, c'est-à-dire peut-être à en attendre moins, à être moins exigeante vis-à-vis d'eux. La correspondance avec Christian P. a sans doute été pour elle un moyen de satisfaire son besoin de s'exprimer, de se raconter, de dialoguer autrement qu'en sollicitant son mari qui n'y semblait pas disposé (il n'en est plus question dans le roman suivant, ce qui pourrait suggérer qu'elle a rempli sa fonction). La nuit avec Igor semble lui avoir permis de se "réveiller", de se retrouver en retrouvant ses sensations de femme. Faire l'expérience que son corps vieillissant «fonctionne» encore, pour reprendre l'expression de Miriam Seidler ${ }^{50}$ lui permet de se « réconcilier » avec lui et de se libérer quelque peu des représentations sociales dominantes qui tendent à "pathologiser» le vieillissement du corps. Une autre auteure, Miriam Haller, évoque également d'autres éléments pour souligner l'importance de cette expérience qu'elle qualifie de « moment de reconnaissance » et qu'elle va même jusqu'à considérer comme le " point culminant du roman " $^{51}$. Selon elle, le caractère transgressif de cette relation d'une femme âgée avec un homme beaucoup plus jeune qu'elle serait aussi typique des «rites de passage» pratiqués dans de nombreuses civilisations pour marquer le passage d'une phase de la vie à une autre ${ }^{52}$. Quant au chien abandonné qu'elle ramasse sur une aire d'autoroute au cours de son trajet de retour, il pourrait avoir pour fonction d'introduire un élément nouveau dans sa vie et de témoigner de sa capacité à accueillir l'inattendu ${ }^{53}$. À la fin du roman, en tout cas, Johanna semble sortie de sa léthargie.

12 Le roman de Monika Maron semble donc pouvoir être rapproché de ce que certains auteurs appellent « roman de la maturation» ( Reifungsroman ») modèle du "Bildungsroman ", dans la mesure où «apprendre à être vieux » serait un 
processus comparable à "apprendre à devenir adulte $"^{55}$ et pourrait relever, selon Miriam Haller ${ }^{56}$, de ce que l'ethnologue Arnold van Gennep appelle "rite de passage " et décrit comme comprenant trois phases: une phase de "séparation", une phase de « marge » et une phase d'« agrégation $»^{57}$ ce qui, appliqué au roman de Monika Maron, pourrait se traduire ainsi : une phase de retrait(e) et de lamentation sur la perte de la jeunesse, une phase de réflexion qui passe notamment par l'écriture des lettres et du journal et une phase de réintégration dans la société avec le retour à Berlin et l'élaboration d'un nouveau projet. Il faudrait donc sans doute distinguer dans la production contemporaine des écrits sur la vieillesse les romans du vieillissement des romans de la vieillesse proprement dits qui concerneraient l'ultime étape avant la mort et non l'entrée dans une nouvelle phase de la vie.

\section{NOTES}

1. Fischer Verlag, Frankfurt am Main, 2002 dont nous abrégerons le titre sous la forme EM. Les numéros de page des citations renvoient à l'édition parue en 2005 au Fischer Taschenbuchverlag. 2. Elle évoque en effet son "soulagement quand elle pouvait dissimuler à nouveau toute sa peau nue de sorte qu'aucun regard ni le [s]ien ni celui de quelqu'un d'autre ne puisse découvrir sur [s]a peau la marque de la vieillesse visible en pleine lumière » («meine Erleichterung [...] wenn ich [...] alle nackte Haut wieder verhüllen durfte, so dass kein zufälliger Blick, weder mein eigener noch der eines anderen, die in grellem Sonnenlicht schon sichtbare Gravur der Greisenhaftigkeit auf meiner Haut entdecken konnte. » EM, 26).

3. «ich hätte [...] feststellen müssen, dass mir schon wieder ein melierter Scheitel gewachsen war» EM, 26.

4. « diesen aussichtslosen Kampf gegen die Haare, die Haut und das Fleisch » EM, 26.

5. « die unbarmherzige tägliche Verwandlung in das ganz alte [Gesicht] », EM, 38.

6. «Ich duschte nicht mehr, sondern badete unter einer Decke aus Schaum [...], ich hasste die unvermeidlichen Augenblicke meiner Nacktheit am Abend und am Morgen. » EM, 27.

7. « Ich kannte längst das Gefühl, wenn die Blicke der Männer mich neutralisierten. » EM, 27.

8. « Enkelkinder sind wohl die einzige natürliche Leidenschaft, die das Alter uns zugesteht. » EM, 176.

9. «Es ist Nacht und ich sehe vieilleicht zu schwarz. » EM, 175-176.

10. « in gewisser Hinsicht war jede Liebe nichts als die Imagination einer paradiesischen Glückseligkeit, deren Urbild in uns irgendwie genetisch verankert sein muss und uns zwingt, ihrer irdischen Erfüllung nachzujagen. » EM, 163.

11. "Elli sagte auch, dass sie in Menschen, die jenseits der triebgesteuerten Jugendzeit ihre Glücksphantasien an die geschlechtliche Liebe hängten, entweder auf den Rausch programmierte Suchtcharaktere, leidverliebte Masochisten oder einfach nur romantische Idioten sehen könne. » EM, 163-164.

12. «In der ländlichen Zurückgezogenheit hoffte ich wiederzufinden, was mich vor zwei Jahren bewogen hatte, dem Verlag mein Vorhaben als einen faszinierenden und originellen Beitrag zur preußischen Geschichte anzupreisen, und was mir ein Jahr später, als der Verlag mir tatsächlich einen Vertrag anbot, verlorengegangen war. » EM 36-37. 
13. Johanna évoque avec des mots très durs « le vide de cette longue période restante au cours de laquelle nous ne sommes plus que des cibles pour vendeurs de tous domaines et des facteurs de coûts colossaux pour les caisses d'assurance maladie »; « diese öde lange Restzeit [...] in der wir nur noch als Zielgruppe von Verkäufern aller Branchen und als katastrophaler Kostenfaktor für die Krankenkassen wichtig sind ", "des êtres inutiles, paresseux " " au point que leurs petitsenfants devront un jour se demander s'ils peuvent les laisser vivre»; «unnütze, faule Menschen », «so dass unsere Enkelkinder eines Tages auf die Idee kommen müssen, ob sie uns überhaupt leben lassen können » EM, 56.

14. «Ich habe jetzt schon das Gefühl, dass ich nichts kann, was diese Welt noch braucht. » EM, 56. 15. «Mein Verlag [...] wurde verkauft und umstrukturiert [...] Die traditionellen Geisteswissenschaften, bisher der Mittelpunkt unseres Programms, sind zum Appendix geschrumpft und werden durch medienwissenschaftliche und bioethische Reihen ersetzt [...] Unser Ressort [...] wurde unters Dach verbannt, wo wir unserer altmodischen Passion nachgehen dürfen, ungestört und ohne zu stören. » EM, 96.

16. «Als sie zum ersten Mal aus Amerika zurückkam, sagte sie, die Deutschen hätten keinen Stolz [...] Die Amerikaner, sagte Laura damals, seien stolz, weil sie ihre Niederlagen genauso verantworten wollten wie ihre Siege. » EM, 174-175.

17. «Vor drei Jahren habe ich zum ersten Mal bemerkt, dass ich erleichtert war, als der Herbst war » EM, 5 et d'ajouter un peu plus loin : «[...] dass ich die Zeit, in der ich den Abschied vom Sommer als eindeutigen Verlust, als Zumutung, sogar als Schmerz empfunden habe, länger als drei Jahre zurückliegen muss, weiß ich genau. » EM, 5.

18. « die ermüdende Fortsetzung des Gewesenen » EM, 138.

19. «Alle wichtigen Kontrakte unseres Lebens haben wir vor langer Zeit geschlossen, auch die falschen, in deren Schlingen wir gefangen bleiben bis zum Ende, wenigstens aber bis zur Rente. Der Beruf, die Anzahl der Kinder, der Wohnort, alles ist endgültig. » EM, 220-221.

20. Johanna emploie le mot terrible de « Restzeit».

21. «Ich fragte mich, ob ich mein Leben, diesen Rest meines Lebens [...], ob ich dieses Leben, wenn es denn möglich wäre, fünfzig oder hundert Jahre länger führen wollte als mir statistisch zustand. » EM, 138.

22. «Ich war schon seit zwei Wochen in Basekow und hatte außer einigen Notizen, die ich zu meiner Lektüre gemacht hatte, nichts geschrieben. » EM, 48.

23. «Ich wusste nicht, was ich denn mit jener Wilhelmine Enke, der Geliebten und Vertrauten des zweiten Friedrich Wilhelm, zu tun haben könnte und schon gar nicht, warum ich eine Biografie über sie schreiben wollte. [...] Überhaupt kam mir alles, für das ich eine gewisse Zuständigkeit vorweisen konnte, seit einiger Zeit belanglos vor. Die Existenz einer Biographie über Wilhelmine Enke war sogar in meinen, ihrer designierten Verfasserin, Augen, vollkommen bedeutungslos, außer für meinen Kontostand. » EM, 37.

24. «Nachdem ich drei Wochen lang nicht nur jede geistige Anstrengung, sondern auch gedankliche Zielgerichtetheit vermieden hatte, drängte es mich noch immer nicht, meinen haustierähnlichen und gänzlich unnützen Zustand zu beenden. Meine anfängliche Hoffnung, etwas in mir würde sich, wenn ich nur lange genug im Stumpfsinn verharrte, auch ohne disziplinierende Vorsätze wehren, erfüllte sich nicht. Im Gegenteil: je länger der Zustand andauerte, umso wohler fühlte ich mich in ihm. » EM, 83.

25. «Sie sollten nicht so über sich sprechen, sagte Igor. Wie so ? So, resigniert. Sonst fühlen Sie sich eines Tages wirklich so. » EM, 234.

26. «Im ersten Jahr nach dem Wunder konnte, wer die Zeichen der Zeit wirklich erkannt hatte und nicht lange zögerte, sein bisheriges Leben schließen wie ein Buch und einfach ein neues beginnen. Achims Zimmernachbar im Institut wurde Minister, nachdem er achtzehn Jahre lang mit der Herausgabe des Gesamtwerkes von Ludwig Tieck befasst war, und der Parteisekretär meines Schallplattenverlags gründete ein Beerdigungsunternehmen. » EM, 41. 
27. «Wahrscheinlich hätte ich damals mit dem Biografienschreiben aufhören sollen. » EM, 41. Cette phrase revient d'ailleurs à plusieurs reprises (EM, 43 ; EM, 213).

28. «Ich hätte alle Festlegungen aufheben und mein Leben neu erfinden dürfen. Ich hätte Blumenbinderin werden können oder Politikerin, Versicherungsvertreterin, ich hätte auch ein Bestattungsunternehmen gründen können [...] », EM, 213.

29. « Ich hatte zum letzten Mal in diesem Jahr den Rasen gemäht und Laub geharkt [...], strich die Außentüren mit Holzschutzmittel ein. Wenn es warm war, saß ich mittags ein oder zwei Stunden in der Sonne, trank Kaffee oder Apfelsaft, sah ziellos über die abgeernteten Felder, verfolgte den müde gewordenen Flug der Wespen oder las den Nordkurier, manchmal auch das kostenlose Werbeblättchen [...] ; abends las ich oder telefonierte [...], sah irgendwelche Serienkrimis [...] und wartete, dass sich in mir etwas rührte, das sich gegen diesen Stumpfsinn wehrte. » EM, 48-49.

30. "wie auf einem Schiff mitten im dunklen Meer, abgeschnitten von den anderen Menschen und allem, was hätte Halt bieten können. » EM, 49.

31. "Ich war schon seit zwei Wochen in Basekow » EM, 48 ; "Nachdem ich drei Wochen lang [...] » EM, 83 ; « in der Woche darauf » EM, 93 ; « am letzten Mittwoch » EM, 223.

32. Lettre à Christian P. (54-57) et lettre non envoyée à Achim (EM, 58), lettre de Christian P. (EM, 93-98), lettre à Christian P. (99-100 ; 111-113), lettre de Christian P. (155-161) lettre à Christian P. (208-217), lettre de Christian P. (217-222); notes sur Wilhelmine Enke (EM, 80-82 ; 103-106; $146-150 ; 195-204)$.

33. «ich führte ein Doppelleben, ein wirkliches und ein erzähltes, wobei sich das eine vom anderen kaum unterschied, nur verstand ich, was ich erlebt hatte, erst, indem ich es erzählte oder mir vorstellte, was geschehen wäre, hätte ich die jeweils andere Entscheidung getroffen. » EM, 91.

34. « Achim langweilten ziellose Gespräche, wie ich sie mit Elli führte », EM, 89.

35. « Er (der Leser) muss begreifen lernen, dass dieses Buch nicht nur das Problem, sondern auch bereits die Lösung des Problems darstellt ». Der Tagesspiegel, 31-8-02.

36. Elle se demande ce qu'Irene aurait choisi si on lui avait donné le choix entre deux possibilités : « ein kurzes, beschädigtes mit einer verbogenen Wirbelsäule, ohne Liebe, ohne Kinder, dafür mit garantiertem beruflichem Erfolg oder gar keins » EM, 137-138.

37. « Achim schrieb immer noch an seiner Dissertation und hätte seine Promotion auf keinen Fall gefährden wollen. Laura würde unter der langen Wartezeit und den damit verbundenen Quälereien leiden. Ich war sicher, dass ich ohne Achim und ohne Laura mit Elli nach Kreuzberg gegangen wäre ». EM, 140.

38. "Solange wir uns kannten, hatte ich Elli immer für erwachsener und lebensklüger, vor allem unabhängiger gehalten als mich. Damals als sie nach Kreuzberg zog, dachte ich, dass Elli im Leben alles richtig macht und ich alles falsch. » EM, 140.

39. "Laura hatte etwas Wildes und Furchtloses an sich [...] Alles Unbändige und Aufbrausende, an dem es mir seit jeher gemangelt hatte [...] bewunderte ich [...]» et de conclure : "In Laura wuchs das Mädchen heran, das ich hätte sein wollen. » EM, 168-169.

40. Elle va jusqu'à disposer une planche pleine de clous à l'endroit, selon elle illicite, où il gare sa voiture et Johanna commente l'incident en notant qu'elle éprouve une sorte de "satisfaction ", «bien que son voisin lui soit indifférent et bien qu'elle ne sache pas s'il a réellement mérité ses pneus crevés. [...] Friedel se défendait et j'aurais bien aimé me défendre, mais je ne savais pas exactement contre quoi, je ne savais pas non plus ce que j'aurais eu à défendre. »: "Ihre lächerliche und unsinnige Nagelbrettfalle erfüllte mich mit Genugtuung, obwohl mir Friedels Nachbar gleichgültig war und ich nicht einmal wusste, ob er die geplatzten Reifen verdient hatte. ... Friedel wehrte sich, und ich hätte mich auch gern gewehrt, nur wusste ich nicht genau, wogegen ich mich wehren oder was ich verteidigen müsste. »EM, 109. Elle va même plus tard, comme le lui raconte une autre femme du village, jusqu'à lâcher son chien contre lui lorsqu'il lui rend visite avec son avocat, EM, 224-227. 
41. «Vor drei Jahren hat Kathrin sich in einen fünfzehn Jahre jüngeren Komponisten verliebt [...] » lui écrit Christian P. en soulignant que cette histoire est « le contraire de toutes les histoires de séparation à nos âges ; d'un point de vie statistique, cela n'existe pas » «Sie (die Geschichte unserer Trennung) ist das Gegenteil aller anderen Trennungsgeschichten in unseren Jahrgängen, statistisch kommt sie gar nicht vor. » EM, 155.

42. «Ich hatte damals in dem Schicksal der Wilhelmine Enke und späteren Gräfin Lichtenau ein Gleichnis gefunden, das nicht nur eine Antwort auf die Verwirrungen meines Lebens zu enthalten schien, sondern etwas Exemplarisches, einen Umkehrschluss oder eine andere Art vertrackter Logik, die mir nicht mehr zugänglich war. », EM, 36

43. EM, 203-204.

44. «Die Enke hat die Chance eines zweiten, unverhofften, ihr durch Geburt nicht zugedachten Lebens, die Friedrich Wilhelm ihr gab, als er in dem dreizehnjährigen Kind die Frau erkannte, die er sich zur Geliebten wünschte, mit allem, was ihr gegeben war, mit Intelligenz, Wissbegier, Schönheit, Willenskraft, gepackt und fortan mit Klauen und Zähnen verteidigt. Alles was dieses geschenkte Leben hergab, hat sie aus ihm gesogen. Sie lernte Sprachen, reiste, eroberte sich die Kunst, Philosophie, Musik, Architektur [...]», EM 215.

45. « Warum dieser Hass gegen sie ? [...] weil sie, als sie längst nicht mehr die Geliebte des Königs war, seine innigste Freundin blieb, und das bis zu seinem Tode? Lag die eigentliche Provokation eben darin, dass diese Geliebte sich sozusagen selbst überlebt hatte, ohne ihren Rang und ihren Einfluss zu verlieren?», EM, 104-105.

46. «Weißt du, dass sie mit fünfzig Jahren [...] den sechsundzwanzigjährigen Theaterdichter Franz von Holbein geheiratet hat? » écrit-elle à Christian P. EM, 216.

47. Ach Glück, Frankfurt, Fischer Verlag, 2007. La genèse de ce livre est le sujet des conférences de poétique que Monika Maron a tenues à l'université de Francfort. Elle y explique que "plus elle réfléchissait à ce à quoi elle voulait s'intéresser au cours des deux ou trois années suivantes, plus il lui apparaissait clairement que ce qu'elle voulait savoir de la manière la plus impérieuse qui soit était ce que faisait Johanna après avoir quitté ses moraines et avoir regagné la ville.» «Je länger ich darüber nachdachte, womit ich mich in den folgenden zwei oder drei Jahren beschäftigen wollte, umso sicherer schien mir, dass ich dringlicher als alles andere wissen wollte, was Johanna, nachdem sie aus den Endmoränen in die Stadt zurückgekehrt ist, tut [...] Ich wollte nicht ein Buch schreiben, weil ich eine mitteilenswerte Geschichte kannte, sondern weil ich herausfinden wollte, wie die Geschichte, die ich in die Welt gesetzt hatte, weitergeht. ", Wie ich ein Buch nicht schreiben kann und es trotzdem versuche, Fischer Taschenbuch Verlag, Frankfurt, 2006, p. 9.

48. Monika Maron a consacré en 2004 un texte à Leonora Carrington et à son roman Das Hörrohr (Le cornet acoustique) "Das Hörrohr von Leonora Carington ", in Doch das Paradies ist verriegelt. Zum Werk von Monika Maron, Hg.v. Elke Gilson, Frankfurt, Fischer Taschenbuch Verlag, 2006, p. 293-295. Elle s'y étonne que ce roman qui met en scène des personnages de vieilles dames quelque peu excentriques et qui lui a beaucoup plu n'ait pas trouvé davantage de lecteurs (il a été traduit en allemand en 1980 et réédité en 1996).

49. Miriam Seidler interprète les réserves des critiques comme le signe que le roman Ach Glück présenterait une nouvelle image de femme vieillissante sortant des schémas traditionnels et encore largement taboue: elle l'oppose notamment à celle du livre de Handke Wunschloses Unglück paru en 1972 soit près de 30 ans plus tôt et dans lequel on trouve une femme âgée semblant n'avoir d'autre issue que la résignation et le suicide. Cf. Miriam Seidler, Figurenmodelle des Alters in der deutschsprachigen Gegenwartsliteratur, Tübingen, Narr Verlag, 2010, p. 118.

50. « dass ihr gealterter Körper funktionstüchtig ist », ibid., p. 109.

51. «Diese Begegnung wird als Moment der Anagnorisis und gleichzeitig als Höhepunkt der Handlung konstruiert - ein Moment der Wiedererkennung der durch ihr Altern sich selbst entfremdeten Johanna ». Cf. Miriam Haller, «Altern erzählen. 'Rites de passage' als narratives 
Muster im zeitgenössischen Roman ", in Soziokulturelle Konstruktionen des Alters. Transdisziplinäre Perspektiven, Hg. v. Dieter Ferring, Miriam Haller, Hartmut Meyer-Wolters, Tom Michels, Würzburg, Königshausen und Neumann, 2008, p. 109.

52. Elle évoque notamment la cérémonie du «kanreki » dans la société japonaise traditionnelle au cours de laquelle les femmes vieillissantes peuvent fumer, boire, se tenir assises les jambes écartées, faire des plaisanteries obscènes avec des hommes plus jeunes, bref faire tout ce qui d'habitude leur est interdit, ibid., p. 102.

53. L'idée du chien renvoie sans doute en partie à une expérience personnelle de Monika Maron qui semble avoir elle-même recueilli un chien abandonné ; elle consacre en tout cas tout son discours de remerciement pour la médaille Carl Zuckmayer à parler de son chien et de l'expérience de « communication sans mots » qu'il lui permet de vivre! « Doch das Paradies ist verriegelt...» Dankesrede zur Carl-Zuckmayer-Medaille, 2003, in Elke Gilson (Hg.), Doch das Paradies ist verriegelt [...], op. cit., p. 270-275.

54. Le terme semble avoir été forgé par Barbara Frey Waxman et est repris notamment par Rosario Arias Dobles dans son article « Moments of Aging. The Reifungsroman in Contemporary Fictions ", in: Brian J. Worsfold (Hg.), Women Aging through Literature and Experience, LLeida 2005, p. 3 et par Miriam Haller «Die 'neuen Alten'. Performative Resignifikation der Alterstopik im zeitgenössichen Reifungsroman", in Dorothee Elm, Thorsten Fitzon, Kathrin Liess, Sandra Linden (Hg.), Alterstopoi. Das Wissen von den Lebensaltern in Literatur, Kunst und Theologie, de Gruyter, Berlin, 2009, p. 237. Rosario Arias Doblas le définit ainsi: «In 1985, Barbara Frey Waxman coined the term Reifungsroman to refer to novels where the female protagonists were forging «new identities or reintegrating fragmented old ones (as well as) acquiring the self-confidence, selfrespect and courage to live the remainder of their lives fully and joyously (Soundings: An interdisciplinary Journal 1985:320)», p. 3.

D'autres auteures anglo-saxonnes, notamment Margaret Morgenroth Gullette et Linda Westervelt (citées par Miriam Seidler, op. cit., p. 118-122) ont proposé les termes de " age novel ", « midlife novel » voire de « midlife Bildungsroman ».

Miriam Seidler soulève à juste titre la question des connotations contenues notamment dans les termes de Reifungsroman et de Bildungsroman qui sous-entendent que l'évolution des protagonistes est nécessairement positive, conduisant par exemple à davantage de sagesse et s'étonne : «Comment l'orientation téléologique du Bildungsroman peut-elle être mise en œuvre dans le cas de protagonistes âgés ? « Wie soll die teleologische Ausrichtung des Bildungsromans im Alter umgesetzt werden? », op. cit., p. 122. Elle propose donc quant à elle le terme plus neutre, mais moins frappant de « Erzählung des mittleren Alters ».

55. Jacques Laforest, Introduction à la gérontologie. Croissance et déclin, éd Frison Roche, Montréal, 1998, p. 76.

56. Op. cit., p. 237.

57. Arnold van Gennep, Les rites de passages, Paris, A. et J. Picard, 1981.

\section{RÉSUMÉS}

L'article s'intéresse au roman de Monika Maron Endmoränen qui évoque l'expérience du vieillissement d'une femme d'une cinquantaine d'années. Il montre comment cette femme qui est aussi la narratrice traverse, pendant un séjour de quelques semaines, seule dans sa maison de campagne, différentes phases qui lui permettent d'envisager un nouveau départ : expérience du 
déclin physique et deuil de la jeunesse perdue; expérience du retrait et réflexion sur sa vie (erreurs, occasions manquées); possible réintégration dans la société. L'article s'interroge également sur l'appartenance générique du roman: Reifungsroman, age novel, midlife novel, midlife Bildungsroman, Erzählung des mittleren Alters.

Der Artikel beschäftigt sich mit Monika Marons Roman Endmoränen, in dem der Alternsprozess einer Mitfünfzigerin thematisiert wird. Er zeigt, wie jene Frau, die auch die Erzählerin ist, während eines mehrwöchigen Aufenthalts in ihrem Landhaus verschiedene Phasen durchläuft, die ihr erlauben, die Möglichkeit eines Neuanfangs zu erwägen: Erfahrung des körperlichen Verfalls und Trauer um die verlorene Jugend ; Erfahrung des Rückzugs und Nachdenken über ihr bisheriges Leben (Fehler, verpasste Gelegenheiten) ; mögliche Reintegration in die Gesellschaft. Anschließend wird noch auf die Frage der Gattungszugehörigkeit des Romans eingegangen: Reifungsroman, age novel, midlife novel, midlife Bildungsroman, Erzählung des mittleren Alters.

The article deals with Monika Marons novel Endmoränen that evokes the ageing process of a woman between 50 and 60 . It shows how the woman who is also the narrator goes through different phases which give her the possibility of envisaging a new beginning: experience of physic decline and mourning of the loss of her youth; experience of retreat and reflexion about her life (failures, missed opportunities), possible reintegration in the society. The article also discusses the question of the generic belonging of the novel: Reifungsroman, age novel, midlife novel, midlife Bildungsroman, Erzählung des mittleren Alters.

\section{AUTEUR}

\section{EMMANUELLE AURENCHE-BEAU}

Université Lumière Lyon 2 